\title{
Adaptive Virtual Reality Games for Rehabilitation of Motor Disorders
}

\author{
Minhua Ma ${ }^{1}$, Michael McNeill ${ }^{1}$, Darryl Charles ${ }^{1}$, Suzanne McDonough ${ }^{2}$, \\ Jacqui Crosbie $^{2}$, Louise Oliver ${ }^{2}$, and Clare McGoldrick ${ }^{2}$ \\ ${ }^{1}$ School of Computing and Information Engineering, \\ ${ }^{2}$ Health and Rehabilitation Sciences Research Institute, \\ University of Ulster, U.K. \\ m.ma@ulster.ac.uk
}

\begin{abstract}
This paper describes the development of a Virtual Reality (VR) based therapeutic training system aimed at encourage stroke patients with upper limb motor disorders to practice physical exercises. The system contains a series of physically-based VR games. Physically-based simulation provides realistic motion of virtual objects by modelling the behaviour of virtual objects and their responses to external force and torque based on physics laws. We present opportunities for applying physics simulation techniques in VR therapy and discuss their potential therapeutic benefits to motor rehabilitation. A framework for physically-based VR rehabilitation systems is described which consists of functional tasks and game scenarios designed to encourage patients' physical activity in highly motivating, physics-enriched virtual environments where factors such as gravity can be scaled to adapt to individual patient's abilities and in-game performance.
\end{abstract}

Keywords: Serious games, Virtual Reality, adaptive games, motor disorder, therapeutic exercise, bilateral training.

\section{Introduction}

The use of VR technology and computer games in motor rehabilitation has been shown in the literature to be an effective way of providing enjoyable and motivating tasks which can be graded to facilitate the rehabilitation process. To date the application of VR to rehabilitation has focused extensively on using the technology to encourage patients to practise active functional tasks in virtual environments. Many studies [1], [2] have identified the benefits of using VR in rehabilitation to improve balance, endurance, dexterity, speed and range of motion.

Currently, most VR applications in rehabilitation simulate real life activities such as grasping and manipulating objects or performing everyday tasks e.g. pick-andplace virtual objects [1], [3], [4], shop in a virtual mall [5], or cross a street [6]. These VR systems help patients enhance improve functional ability and realise greater participation in community life. However, object animations in most VR therapy systems are pre-rendered and hence not realistic in terms of physical simulation. 


\subsection{Physically-Based Simulation}

Physically-based simulation creates realistic motions of virtual objects based on the laws of physics. The behaviour of virtual objects and their responses to external force and torque are simulated in a physically realistic manner. Physics simulation models objects with physical properties, such as mass, inertia, barycentre, joint limitations, restitution and surface friction - it can make objects in virtual worlds not only look real but act real. Typical dynamics simulation includes collision detection and the simulation of gravity, friction force, torque, and kinematics in motor actions. Physics can be applied to rigid bodies or deformable bodies such as human tissues. Rigid body dynamics plays an important role in VR motor rehabilitation and is especially needed when dextrous manipulation of virtual objects is concerned.

The advantage of using VR in clinical settings is that by virtue of its programmability, environments and the type and difficulty of tasks can be modified according to the user's capacities and therapeutic goals. Physics simulation provides more flexibility on experiment configuration in which not only object positions, orientations, size can be modified but also gravity, restitution, force and torque damper, and joint limitations can be reliably modified [7], thereby creating a very individualised set of rehabilitation tasks which would be impossible to achieve with the user's residual motor control in the physical world. These tasks can then be reliably recreated over a period of several weeks or months and patient outcome can be assessed. The addition of physics to the VR therapy may increase patients' experience of immersion in virtual environments, which may in turn increase engagement and activity enjoyment and thereby improve clinical outcome.

The quality of physics that is required in a simulation is application specific. In the cognitive and affective domains of learning where the focus of training is more on attitudes, high physical fidelity is not always required. However, in medical simulation such as surgery planning and training, high quality physical fidelity is so important that without it the skills acquired in the virtual world may not transfer to the real one. Recent research suggests that physics fidelity may be important for motor rehabilitation. Viau et al. [1] compared movement kinematics of identical tasks of reaching, grasping, transporting, placing and releasing a ball in a real and a virtual environment. Their results showed that there is a slight change in motor patterns when grasping and placing a ball in the two reality conditions for both healthy subjects and hemiparesis patients with motor deficits, which was mainly due to lack of collision handling in the VE. Their interpretation of the results is that both healthy subjects and individuals with motor deficits used similar movement strategies in pick-and-place tasks in the two conditions, and they concluded that training of arm movements in VR may be a valid approach to the rehabilitation of patients with motor disorders. However, the results also suggest that physics simulation in VR rehabilitation may reduce the difference of movement strategies in the virtual and real world, which may be an important factor in the transferability of virtual skills to the real world.

\section{Adaptive Rehabilitation}

Ideally, rehabilitation should be targeted to the individual needs of patients. Patients have a wide range of abilities and tasks which are impossible for some can be trivial 
for others. It is usual to tailor a stroke rehabilitation session to individual patients according to the type of injury (left/right hemiplegia) and their capabilities. Typically this is done by assessing the patient in a number of standardised tests prior to the rehabilitation session. The therapist can then create a suitably challenging set of exercises for the patient. A key principle in rehabilitation is matching therapeutic tasks to the patients' abilities in order to enable them to improve residual capabilities without causing fatigue and frustration. This is a time-consuming part of the physiotherapist's duties, as constant monitoring is required to ensure the tasks remain adequately challenging throughout the sessions, which typically last for several weeks.

\subsection{Adaptivity}

Adaptation is one technique that VR therapy systems can exploit to benefit a group of users with a wide range of abilities. In order to maintain patient motivation, rehabilitation tasks should be set at an appropriate level of challenge. Also, for the patient to stay engaged in the process, he or she should experience a feeling of immersion in the virtual environment. Charles et al. [8] applied flow theory to adaptive game design, and they suggested that the game should find a balance between the annoyance of an activity that is perceived as trivial and the frustration of one that is perceived as too difficult. This is even more important in the context of VR motor rehabilitation due to the repetitive nature of the exercises and the limitations of the users (patients).

A physiotherapist will typically design motor therapy exercises for a patient based on a number of factors, including the patient's age, gender, culture background, usual handedness, and his or her medical condition (e.g., time since stroke, left or right hemiplegia and cognitive, sensory, and motor abilities based on standardised tests such as the Line Cancellation Test). Not only the difficulty level of the exercises but also the body parts activated during the task may be adapted to individual patient's needs. For example, tasks may be developed to train a specific movement such as wrist extension in order to increase range of motion or endurance of the wrist joint. This data, taken together, forms an individual patient profile which can then be used to initially configure the system to present a suitably challenging, individualised rehabilitation session. Various elements of the simulation can be configured from this profile data, including the number and type of objects, their sizes, speed, mass, distance between objects and the patient, distance of object transportation etc. Additionally, data collected during the rehabilitation session can be used to further improve the patient experience. Such in-session data (e.g., the time taken to perform a task or achieve a goal in a game, the accuracy rate or stimuli-response time) can be used to evaluate the initial configuration of the system. If tasks are not being successfully completed then the objects or game elements in the simulation should be configured to make them easier. Objects can be made larger (easier to grasp), for example, or moved closer to the patient (easier to reach). Alternatively, if activities are being completed much too quickly it may be that the activity is too easy for the patient, and corresponding changes can be made to make the task more challenging.

The ability to dynamically adjust the difficulty of the simulation is a key benefit since in addition to offering a tailored solution which suits the patient's individual needs it also decreases the dependence on human therapists to monitor and provide similar solutions. 


\subsection{User Profiling}

We have constructed a patient model using traditional symbolic classifiers based on patients' age, time since stroke, left/right hemiplegia, impairment and functional measurement, and hemispatial neglect. Table 1 below shows the profile of three patients participating in the study. For each patient, three motricity index and ARAT scores are given, representing their status before training, post training, and 6 weeks after training. Patients 1 and 3 have no hemispatial neglect, while patient 2 has a mild deficit. Table 2 shows how we use patient profile to setup parameters in VR simulations initially.

Table 1. Examples of patient profiling: Motricity Index [9] is for impairment measurement, and Action Research Arm Test (ARAT) [10] is for functional measurement

\begin{tabular}{cccc}
\hline & Patient \#1 & Patient $\# 2$ & Patient \#3 \\
\hline Age & 76 & 62 & 42 \\
Gender & $\mathrm{M}$ & $\mathrm{F}$ & $\mathrm{M}$ \\
Years since stroke & 4 & 4 & 1 \\
Usual handedness & $\mathrm{R}$ & $\mathrm{R}$ & $\mathrm{R}$ \\
Left/Right hemiplegia & $\mathrm{L}$ & $\mathrm{L}$ & $\mathrm{L}$ \\
Motricity Index & $77,77,81$ & $62,73,77$ & $77,79,77$ \\
ARAT & $3,6,11$ & $4,11,19$ & $54,53,54$ \\
Hemispatial neglect & None & Left-sided & None \\
\hline
\end{tabular}

\section{A Framework for VR Motion Therapy}

Fig. 1 shows our framework for VR post-stroke motor rehabilitation. Rehabilitation tasks consist of functional tasks, such as wrist extension, reaching, grasping and catching, and game simulations. The system allows users to interact with virtual objects in real-time through multiple modalities and to practice specific motor skills. Users of the system are stroke patients with motor deficits and physiotherapists who, as operators, are responsible for initialising the system and controlling the scripting of tasks. Input devices include ordinary devices, mouse and keyboard for operators and a range of real-time motion tracking devices - two 5DT Ultra DataGloves which capture finger flex and hand postures (Fig. 2c); Four Ascension MotionStar wireless magnetic sensors (Fig. 2a) are used to track the patient's hand, arm and upper body movements.

Output involves visual, audio and haptic modalities. The dual output visual interface includes a desktop monitor for operators and a high resolution head-mounted display (HMD) equipment, Virtual Research 1280 stereo (Fig. 2b), for patients. Haptic interface is a possible extension of the system but it is not our current concern.

The device drivers interface the specialised hardware with the software components which include a 3D graphic engine based on OGRE [11], dealing with loading of mesh data, rendering, scene management and the user interface, and a rehabilitation engine which creates functional (task-based) or non-functional 
Table 2. Initial setup of parameters of VR therapy based on patient profile

\begin{tabular}{|c|c|c|}
\hline Impairment measures & Parameters of VR therapy & Goals \\
\hline $\begin{array}{l}\text { Motricity Index } \\
\text { (Elbow flexion score, } \\
\text { Shoulder abduction score) } \\
\text { ARAT (gross movement score) }\end{array}$ & $\begin{array}{l}\text { Size of target area on } x-z \text { plane (initial } \\
\text { coordinates of falling oranges in the } \\
\text { catching task), range of mice positions in } \\
\text { the whack-a-mouse game }\end{array}$ & $\begin{array}{l}\text { Range of movement } \\
\text { Gross movement }\end{array}$ \\
\hline ARAT & Size of oranges in the catching task & Gross movement, accuracy \\
\hline ARAT & Size of basket in the catching task & Gross movement, accuracy \\
\hline $\begin{array}{l}\text { Hemispatial neglect } \\
\text { L/R hemiplegia } \\
\text { Usual handedness }\end{array}$ & $\begin{array}{l}\text { Loci of oranges in the catching task, } \\
\text { Loci of mice and dogs in the whack-a- } \\
\text { mouse game }\end{array}$ & $\begin{array}{l}\text { Movement of affected arm } \\
\text { Attention of affected side }\end{array}$ \\
\hline Motricity Index (pinch grip score) & Size of objects (cube, ball, cylinder, cup) in & Accuracy, dexterity \\
\hline ARAT (grasp, grip, \& pinch score) & the pick-and-place task & Hand/finger movement \\
\hline $\begin{array}{l}\text { Motricity Index (elbow flexion } \\
\text { score, shoulder abduction score) } \\
\text { ARAT }\end{array}$ & $\begin{array}{l}\text { Gravity in the catching task, } \\
\text { Length of time that the mouse is stationary } \\
\text { in the whack-a-mouse game }\end{array}$ & $\begin{array}{l}\text { Speed } \\
\text { Accuracy }\end{array}$ \\
\hline $\begin{array}{l}\text { Motricity Index (elbow flexion } \\
\text { score, shoulder abduction score) } \\
\text { ARAT (gross movement score) } \\
\end{array}$ & $\begin{array}{l}\text { Time between falling oranges in the } \\
\text { catching task }\end{array}$ & Speed \\
\hline
\end{tabular}

(game-based) activities. We use Ageia PhysX [12] to handle dynamics simulation, e.g., articulated structures, robust $\mathrm{CD}$, and friction/contact modelling. A wrapper called NxOgre [13] is used to integrate PhysX with OGRE. The databases consist of mesh data of $3 \mathrm{D}$ models and patient profiles which may be used to automatically adapt the tasks to specific therapeutic needs.

The dynamic adaptation part of the system uses patient profile data and patient progress data to select tasks and initially configure the difficulty level of the tasks and the game. This continues in-session, as all patient interaction with the system is recorded and analysed for in-game adaptation. Hence the system can provide data on progress for individuals that may be collated across the recovery period or across individuals to provide a database on patient progress. The data analysis module enables visualization of patients' movement trajectories and displays joint angles, range of movement and velocities. Currently this occurs off-line, after the rehabilitation session has finished.

\subsection{Functional Training}

Previous study shows that pyramidal tract (e.g. fingers, lower limb) is often impaired after stroke, whereas more proximal areas may be less profoundly affected. [14] It is therefore common for people with stroke to exhibit problems with manipulative movements, on the contra-lateral side, following unilateral stroke. The functional training part of the VR therapy focuses on manipulative movements of fingers, hands and arms such as reaching, grasping, placing, and wrist extension. The 3D position and orientation of the user's hand, elbow, and shoulder are sampled via using three magnetic sensors. Sensor 1 is placed on the volar aspect of the hand, at the base of the head of the 3rd metacarpal bone, Sensor 2 is placed on the posterior aspect of the elbow, just above the olecrannon process, and Sensor 3 is placed on the anterior aspect of the shoulder, on the greater tuberosity of the humerus. 


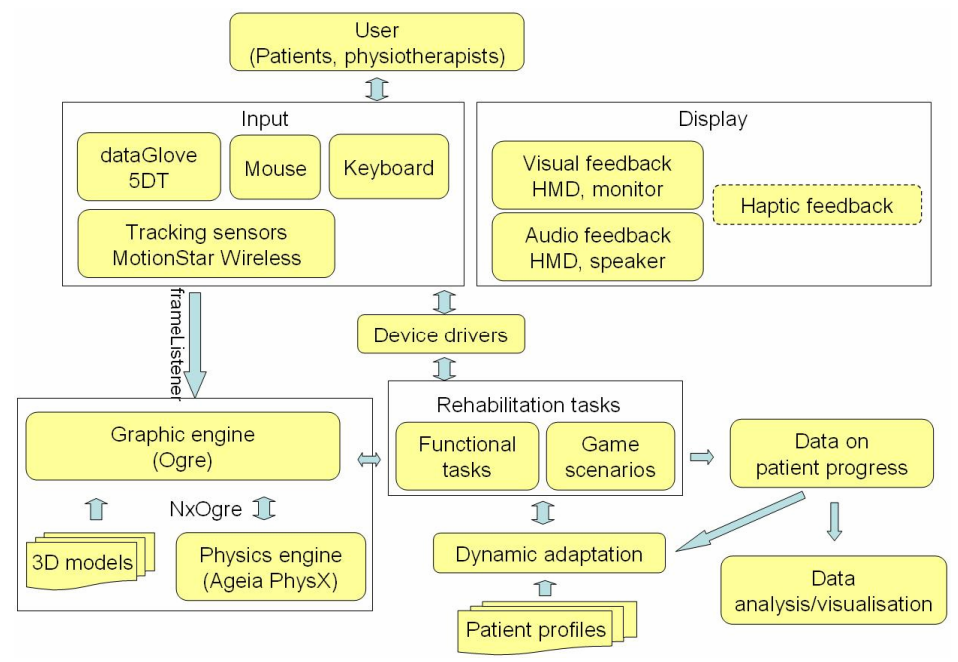

Fig. 1. A framework for physically-based VR rehabilitation

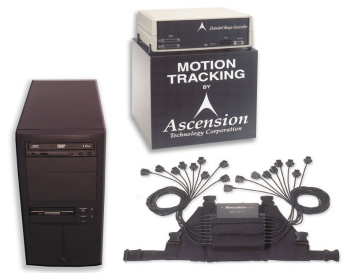

a. MotionStar wireless

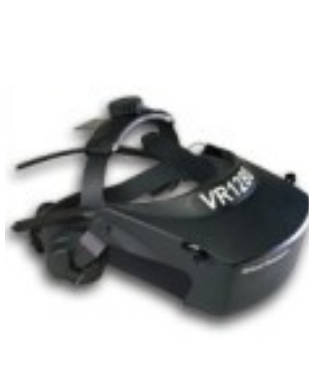

b. VR 1280 HMD

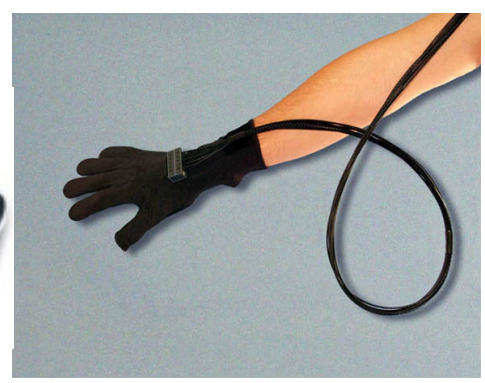

c. 5DT data glove

Fig. 2. Hardware

In the functional training part we simulate a virtual kitchen where subjects are asked to manipulate virtual objects of various shapes and sizes at different locations, such as a typical pick-and-place task by reaching and touching a start marker, grasping a virtual cup, and placing it on a saucer some distance away. When the virtual hand, controlled by the tracking sensors and data glove, intersects with any object in the VE, an auditory signal is played and the object changes colour to indicate success (collision between hand and object detected). Patients use the hand of their hemiplegia side to perform the task.

\subsection{Bilateral Training}

Traditional therapies of upper limb rehabilitation following stroke primarily focus on unilateral strengthening exercises and functional training of the paretic limb. Recent studies in neuroplasticity and stroke rehabilitation have suggested that bilateral 
training may prove beneficial [15], by activating similar neural networks in both hemispheres when voluntary movements of the intact limb are initiated. A catching task has also been developed, where the user holds a virtual basket with either one hand or two (for bilateral upper limb training) to catching falling oranges which fall at random onto a target area (Fig. 3). The position/orientation of the virtual basket is controlled by a sensor attached either on the user's hand if one hand is used to perform the task, or on a real object, a real basket, for example, which the user holds with both hands. If the patient tilts the basket, he or she may not able to catch the oranges and the ones already in the basket may fall to the ground. The target area on the X-Z plane, the falling speed of oranges (controlled by simulating gravity in the virtual environment), the time between oranges falling and the size of oranges and the basket can all be adjusted to suit individual patient's needs. All sensor data is recorded to disk, allowing post-therapy visualisation of the trajectories and analysis of joint angles, velocity, acceleration and range of movement.

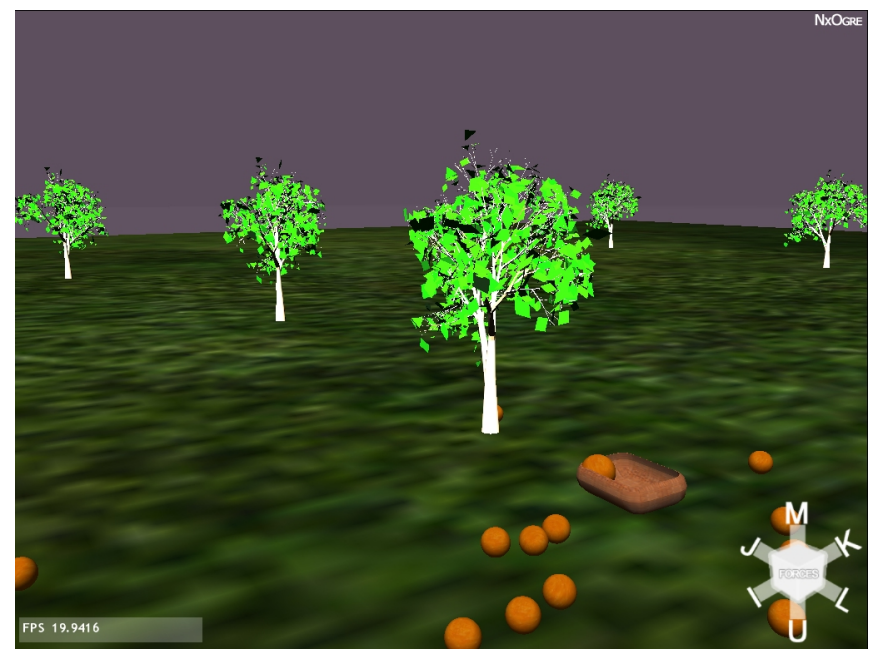

Fig. 3. A functional catching task

\subsection{An Adaptive Game for Motor Rehabilitation}

We have developed an adaptive VR "whack-a-mouse" game for post-stroke therapeutic exercises as shown in Fig. 4. The aim of this game is to encourage gross movement and to improve accuracy and speed of users' upper limb movement. It has also been designed to improve patient's visual discrimination and selective attention, important aspects of stroke rehabilitation where the patient exhibits hemi-spatial neglect. The mouse appears at a random location on the table top, stays there for a certain number of seconds (the actual time it stays still depends on the patient profile), and then re-appears on another part of the table. While the mouse is stationary the patient tries to hit it using a virtual hammer which is controlled by the position and orientation of sensor 1 on the patient's hand. This simulation encourages faster, grosser and less accurate movement than the functional tasks described earlier. 


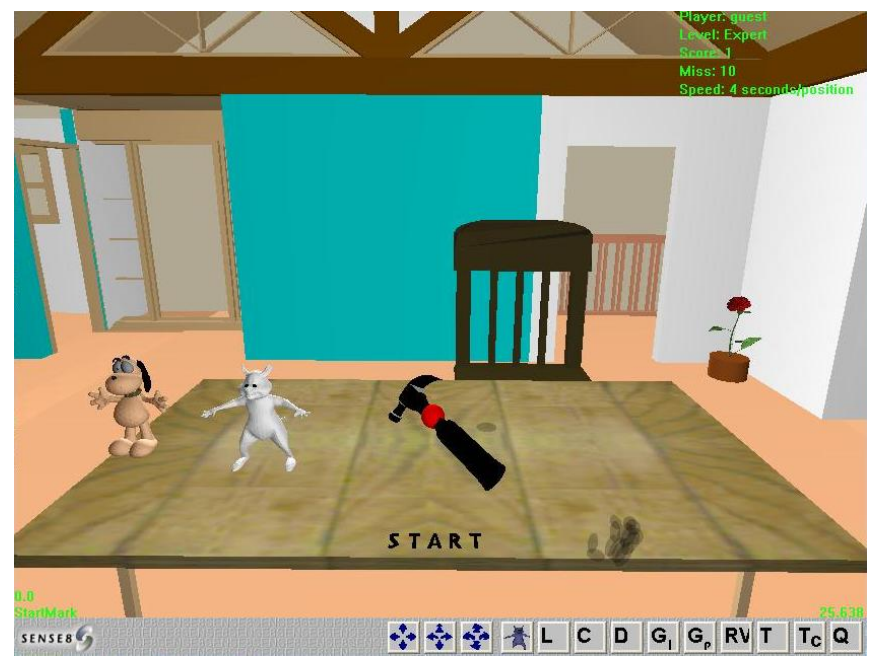

Fig. 4. A 'whack-a-mouse' game

Initial configuration of the game is done automatically based on the profile of the player. For example, the game will be configured to be easier for patients with serious upper extremity motor impairment, i.e., the mouse will stay still for a longer period of time for them than for patients with minor motor impairment. The locus of stimuli will also depend on if a) the patient has right or left-sided hemiplegia and b) if the patient exhibits hemi-spatial neglect. For left-sided hemiplegia patients, the random positioning of the mouse is biased towards the right; similarly for the left. If the patient also exhibits hemi-spatial neglect this bias can be increased. It is important that patients are given a chance to familarise themselves with the game and not be presented initially with something that is too difficult, which could lead to disengagement. Therefore we tend to be conservative with the initial setup.

Patients will learn as they play and their progress of learning may be different. Therefore, the configuration that we use at the beginning of the game may no longer be appropriate as the game proceeds. The length of time that the mouse is stationary and the locus of mouse and dog adapts dynamically to patient performance. The mouse appears more frequently in the quadrant where the patient has more misses and seems to have difficulty due to either motor deficit or attention deficit.

There are, therefore, a number of adaptive elements of the game which change dynamically according to how well or badly the user is performing. The game has been programmed to enable automatic progression between three levels (Beginner, Intermediate and Expert) under preset conditions. At the Beginner level the time that the mouse is stationary is set automatically according to the patient profile. At the Intermediate level, the time adapts to the patient's performance (to begin with, the speed is the same as in the beginner level). The game progresses according to a simple accuracy test based on the number of mice hit and the number missed, where

miceHit

$$
\text { accuracy }=\text { miceHit }+ \text { miceMissed }
$$


When the accuracy rate drops below a certain threshold, the length of time that the mouse remains still for increases which has the effect of slowing the game, making it easier; and when the accuracy rate exceeds a certain threshold, this time decreases, making the game harder. The Expert level contains both a mouse and a dog which appear simultaneously: patients must hit as many mice as possible and try to avoid hitting the dog. Hitting a mouse increases the score by one, but hitting a dog decreases it by one. This aspect to the game forces patients to search for and hit only the mouse when both mouse and dog are present, in order to improve visual discrimination and selective attention, which can be important aspect to patient rehabilitation particularly for patients with hemispatial neglect.

The player, level of game, score, and adapted speed are shown in the upper-right corner of the screen to give the user real-time feedback. Overall performance data such as score, accuracy rate, and the length of game-play sessions are also shown to the user when the game is over or on entry to the next level, and this data is also written to a session log file for the user. The next time the patient uses the system this information will be used to configure the game. Further, the physiotherapist can analyse this data to assess how the patient is progressing over a number of sessions. Besides the performance feedback, multiple sensory feedback is given. When a mouse is hit, it squeaks and its colour changes to red, and when a dog is hit, it barks and its colour changes to red. To create an element of competition, but also to give feedback to the patient, the user can view the highest scores of all the users and also his or her personal best scores of each game level.

\section{Conclusion}

This paper has shown how adaptive VR games can contribute to motor therapy by providing motivating tasks that can automatically adapt to individual patient's capabilities. We discussed the potential for the integration of physics simulation techniques into VR games for upper limb motor rehabilitation. A physically based VR framework with adaptive game configuration based on data collected before-game and in-game was described which is currently being used by a number of hemiplegia patients with various levels of severity. The integration of physically-based simulation with conventional clinical therapy and game-like scenarios adds richness to the virtual environment which has the potential for improving patient outcome. Initial feedback is very positive, with patients reporting that they enjoy playing the game. Further work is necessary to determine just how much the introduction of physics into the game simulation actually influences patient outcome, but we believe that adaptive game technology has much to offer motor rehabilitation.

\section{References}

1. Viau, A., Feldman, A.G., McFadyen, J., Levin, M.F.: Reaching in reality and virtual reality: a comparison of movement kinematics in healthy subjects and in adults with hemiparesis, Journal of NeuroEngineering and Rehabilitation, 1.11 (2004)

2. Holden, M.K., Dyar, T.A., Schwamm, L., Bizzi, E.: Virtual Environment-based Telerehabilitation in Patients with Stroke, Presence - Virtual Rehabilitation, M. Alexa and J. Marks (Eds), 14.2, pp. 214-233 (2005) 
3. Kuttuva, M., Boian, R., Merians, A., Burdea, G., Bouzit, M., Lewis, J., Fensterheim, D.: The Rutgers Arm: An Upper-Extremity Rehabilitation System in Virtual Reality, Proc. of the 5th International Workshop on Virtual Rehabilitation (IWVR05), Catalina Island, California, U.S.A, pp. 94-103 (2005)

4. McNeill, M.D.J., Pokluda, L., McDonough, S., Crosbie, J.: Immersive virtual reality for upper limb rehabilitation following stroke, IEEE International Conference on Systems, Man and Cybernetics, October pp. 2783-2789 (2004)

5. Rand, D., Katz, N., Shahar, M., Kizony, R., Weiss, P.L.: The virtual mall: development of a functional virtual environment for stroke rehabilitation, Abstracts of the 55th Annual Conference of the Israeli Association of Physical and Rehabilitation Medicine (2004)

6. Katz, N., Ring, H., Naveh, Y., Kizony, R., Feintuch, U., Weiss, P.L.: Interactive Virtual Environment training for safe street crossing of right hemisphere stoke patients with unilateral spatial neglect, ICDVRAT 2004: Proceedsings of the Fifth International Conference on Disability, Virtual Reality and Associated Technologies, Oxford, UK (2004)

7. Ma, M., McNeill, M., McDonough, S., Crosbie, J., Oliver, L.: Physics Fidelity of Virtual Reality in Motor Rehabilitation. Virtual: a Real Success: Proc. Of the 8th International Conference on Virtual Reality (VRIC_-Laval Virtual), S. Richir and E. Klinger (Eds.), pp. 35-41, Laval, France, (April 2006)

8. Charles, D., McNeill, M., McAlister, M., Black, M., Moore, A., Stringer, K., Kücklich, J., Kerr, A.: Player-centred game design: player modelling and adaptive digital games, DIGRA2005: Proceedings of the Second International Digital Games Research Conference, pp. 285-298, Vancouver, Canada, (June 2005)

9. Demeurisse, G., Demol, O., Robaya, E.: Motor evaluation in vascular hemiplegia. European Neurology 19, 382-389 (1980)

10. Lyle, R.C.A.: Performance test for assessment of upper limb function in physical rehabilitated treatment and research. Int. J Rehabil 4, 483-492 (1981)

11. OGRE: Object-oriented Graphics Rendering Engine (2007), http://www.ogre3d.org

12. Ageia: Ageia PhysX SDK (2007), http://www.ageia.com

13. NxOgre (2007), http://www.nxogre.org

14. Smyth, M., Wann, J.: Interactive interfaces for movement rehabilitation in virtual environments, Proceedings of the third International Conference Series on Disability, Virtual Reality and Associated Technologies (ICDVRAT), Alghero, Sardinia, Italy (September 2000)

15. Lewis, G., Byblow, W.: Neurophysiological and behavioural adaptations to bilateral training intervention in individuals following stroke. Clinical rehabilitation 18, 48-59 (2004) 